\title{
Enhanced 3-Sulfanylhexan-1-ol Production in Sequential Mixed Fermentation with Torulaspora delbrueckii/Saccharomyces cerevisiae Reveals a Situation of Synergistic Interaction between Two Industrial Strains
}

OPEN ACCESS

Edited by:

Alberto Mas,

Rovira i Virgili University, Spain

Reviewed by: Kalliopi Rantsiou, University of Turin, Italy Aspasia Nisiotou, Technological Educational Institute of Athens, Greece

Manuel Ramírez,

Universidad de Extremadura, Spain

*Correspondence:

Marina Bely marina.bely@u-bordeaux.fr

Specialty section: This article was submitted to

Food Microbiology,

a section of the journal

Frontiers in Microbiology

Received: 03 November 2015

Accepted: 23 February 2016

Published: 15 March 2016

Citation:

Renault P, Coulon J, Moine V,

Thibon C and Bely M (2016) Enhanced 3-Sulfanylhexan-1-ol Production in Sequential Mixed

Fermentation with Torulaspora delbrueckii/Saccharomyces cerevisiae Reveals a Situation of Synergistic Interaction between Two Industrial Strains. Front. Microbiol. 7:293. doi: 10.3389/fmicb.2016.00293

\author{
Philippe Renault ${ }^{1,2}$, Joana Coulon ${ }^{2}$, Virginie Moine ${ }^{2}$, Cécile Thibon ${ }^{1,3}$ and Marina Bely ${ }^{1 *}$ \\ 1 Unité de Recherche CEnologie, EA 4577, Institut des Sciences de la Vigne et du Vin, University of Bordeaux, Villenave \\ d'Ornon, France, ${ }^{2}$ BioLaffort, Bordeaux, France, ${ }^{3}$ Unité de Recherche CEnologie, USC 1366, Institut des Sciences de la \\ Vigne et du Vin, Institut National de la Recherche Agronomique, Villenave d'Ornon, France
}

The aim of this work was to study the volatile thiol productions of two industrial strains of Torulaspora delbrueckii and Saccharomyces cerevisiae during alcoholic fermentation (AF) of Sauvignon Blanc must. In order to evaluate the influence of the inoculation procedure, sequential and simultaneous mixed cultures were carried out and compared to pure cultures of $T$. delbrueckii and $S$. cerevisiae. The results confirmed the inability of T. delbrueckii to release 4-methyl-4-sulfanylpentan-2-one (4MSP) and its low capacity to produce 3-sulfanylhexyl acetate (3SHA), as already reported in previous studies. A synergistic interaction was observed between the two species, resulting in higher levels of 3SH (3-sulfanylhexan-1-ol) and its acetate when S. cerevisiae was inoculated $24 \mathrm{~h}$ after T. delbrueckii, compared to the pure cultures. To elucidate the nature of the interactions between these two species, the yeast population kinetics were examined and monitored, as well as the production of $3 \mathrm{SH}$, its acetate and their related nonodorous precursors: Glut-3SH (glutathionylated conjugate precursor) and Cys-3SH (cysteinylated conjugate precursor). For the first time, it was suggested that, unlike S. cerevisiae, which is able to metabolize the two precursor forms, T. delbrueckii was only able to metabolize the glutathionylated precursor. Consequently, the presence of T. delbrueckii during mixed fermentation led to an increase in Glut-3SH degradation and Cys-3SH production. This overproduction was dependent on the T. delbrueckii biomass. In sequential culture, thus favoring T. delbrueckil development, the higher availability of Cys-3SH throughout AF resulted in more abundant 3SH and 3SHA production by S. cerevisiae.

Keywords: non-Saccharomyces, Torulaspora delbrueckii, wine, fermentation, mixed inoculation, volatile thiols, aroma precursors 


\section{INTRODUCTION}

Volatile thiols are powerful aromatic compounds that contribute to the fruity notes of many white wines, especially Sauvignon Blanc. The three most important thiols in Sauvignon Blanc aroma are considered to be 3-sulfanylhexan-1-ol (3SH, formerly known as 3MH; Tominaga et al., 1998a), its acetate, 3-sulfanylhexyl acetate (3SHA, formerly known as 3MHA; Tominaga et al., 1996), and 4-methyl-4-sulfanylpentan-2-one (4MSP, formerly known as 4MMP; Darriet et al., 1995; Tominaga et al., 1996, 1998a). Descriptors such as box tree and broom for 4MSP and grapefruit/passion fruit for $3 \mathrm{SH}$ match the occurrence of these compounds in box tree and yellow passion fruit, respectively (Tominaga and Dubourdieu, 1997, 2000). Due to their low perception thresholds (a few $\mathrm{ng} / \mathrm{L}$ ), they contribute significantly to the aroma profile of many wines (Roland et al., 2011 and cited references).

The release of volatile thiols by Saccharomyces cerevisiae yeast during alcoholic fermentation (AF), now relatively well described, results from the biotransformation of non-odorous precursors present in grapes (Tominaga, 1998; Marullo and Dubourdieu, 2010). 4MSP and $3 \mathrm{SH}$ are produced from cysteinylated (Cys-4MSP, Cys-3SH) and glutathionylated (Glut4MSP, Glut-3SH) conjugates by yeast $\beta$-lyase cleavage (Darriet et al., 1995; Tominaga et al., 1998b; Peyrot des Gachons et al., 2002; Subileau et al., 2008a; Fedrizzi et al., 2009; Roland et al., 2011; Coetzee and du Toit, 2012). The biotransformation of these precursors by yeast involves their uptake through the membrane, followed by cleavage into their corresponding aromas (for a review, see Coetzee and du Toit, 2012). Concerning 3SH, in S. cerevisiae, the cysteinylated precursor form is taken up by amino acid transporters, such as Gap1p (Subileau et al., 2008b), while the glutathionylated form is assimilated through the Optlp GSH transporter (Subileau et al., 2008a). Once transported into the cytoplasm, these precursors are transformed by $\alpha, \beta$-elimination, catalyzed by $\beta$-lyases (Howell et al., 2005; Thibon et al., 2008; Holt et al., 2011; Roncoroni et al., 2011; Cordente et al., 2015). However, biotransformation rates by S. cerevisiae are low, with calculated yields ranging from $<1 \%$ to about 5\% (Murat et al., 2001; Dubourdieu et al., 2006; GrantPreece et al., 2010; Kobayashi et al., 2010; Winter et al., 2011). $3 \mathrm{SHA}$ is produced after $3 \mathrm{SH}$ release by alcohol acetyltransferase, encoded by the ATF1 gene in S. cerevisiae (Swiegers et al., 2006). The final concentration of 3SHA depends on the activity balance between alcohol acetyltransferase (promoting esterification of the corresponding alcohol) and esterase (promoting its hydrolysis), encoded by the IAH1 gene (Coetzee and du Toit, 2012).

In recent years, several authors have highlighted the positive contribution of non-Saccharomyces yeasts to the analytical and sensory composition of wine, leading to the commercialisation of certain non-conventional yeasts. This is the case of the Torulaspora delbrueckii species, now available as an active dry yeast. Indeed, this species has been described as having a positive impact on the organoleptic quality of wines, due to its low production of compounds such as acetic acid, ethyl acetate, acetaldehyde, acetoin, hydrogen sulfide and volatile phenols, hence minimizing off-flavors (Cabrera et al., 1988; Herraiz et al.,
1990; Martinez et al., 1990; Ciani and Picciotti, 1995; Ciani and Maccarelli, 1998; Shinohara et al., 2000; Plata et al., 2003; Renault et al., 2009). A strong $\beta$-glucosidase activity, which enhances wine aroma by hydrolysing terpenyl-glycosides, was also described in several T. delbrueckii strains (King and Richard Dickinson, 2000; Hernandez-Orte et al., 2008; Comitini et al., 2011; Azzolini et al., 2012). Moreover, overall, T. delbrueckii alone produced lower quantities of esters than S. cerevisiae (Viana et al., 2008; Sadoudi et al., 2012) but a few minor esters (ethyl propanoate, ethyl isobutanoate, and ethyl dihydrocinnamate) were produced in larger concentrations, which had a positive organoleptic impact on the wine (Renault et al., 2015).

Despite a good ethanol production (up to $11 \%$ vol ethanol) compared to other non-Saccharomyces yeasts (Cabrera et al., 1988; Herraiz et al., 1990; Ciani and Picciotti, 1995; Ciani and Maccarelli, 1998; Renault et al., 2009; Velázquez et al., 2015), T. delbrueckii alone cannot complete AF under winemaking conditions. T. delbrueckii/S. cerevisiae multi starters have thus been proposed to modulate wine flavor and properties and to ensure complete AF. An increasing number of studies using these mixed cultures have, however, produced contradictory results concerning their impact on wine quality. In fact, the inoculation procedure, as well as the different strains used, drastically impact the population dynamics of both species, thus modifying aroma production (Renault et al., 2015).

Few researchers have investigated volatile thiol formation by the $T$. delbrueckii yeast metabolism in pure and mixed cultures. According to Zott et al. (2011), in synthetic medium, T. delbrueckii released significant concentrations of $3 \mathrm{SH}$ but lower than that of pure $S$. cerevisiae cultures. It also has a poor capacity to form 4MSP. These results were confirmed by Sadoudi et al. (2012) during AF of Sauvignon Blanc must. As a result, in a simultaneous mixed culture, at a 10:1 ratio ( $T$. delbrueckii/S. cerevisiae), a decrease in $3 \mathrm{SH}$ and 3SHA production was observed, compared to a pure S. cerevisiae culture.

This study compared the volatile thiol profiles of Sauvignon Blanc wines fermented with pure S. cerevisiae and T. delbrueckii, as well as mixed cultures (simultaneous and sequential). To elucidate the nature of the interactions between these two yeast strains, $3 \mathrm{SH}, 3 \mathrm{SHA}$, their related precursors, and $4 \mathrm{MSP}$, as well as the population dynamics, were monitored throughout AF.

\section{MATERIALS AND METHODS}

\section{Yeast Strains}

In this study, two commercial strains from Laffort company (France) were used: S. cerevisiae Zymaflore ${ }^{\circledR} \mathrm{X} 5$ and T. delbrueckii

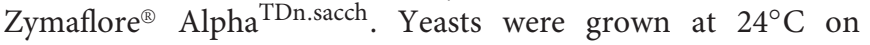
complete YPDA medium ( $1 \%$ yeast extract, $1 \%$ peptone, $2 \%$ dextrose) solidified with $2 \%$ agar, adjusted to $\mathrm{pH} 4.8$.

\section{Fermentation Medium}

The medium used was a Sauvignon Blanc grape must from Bordeaux area, $\mathrm{pH}: 3.15$, with a sugar concentration of $203 \mathrm{~g} / \mathrm{L}$ and an available nitrogen concentration adjusted to $210 \mathrm{mg} / \mathrm{L}$ 
(i.e., amino acids: $114 \mathrm{mg} / \mathrm{L}$ and ammonia: $96 \mathrm{mg} / \mathrm{L}$ ). The total and free sulfur dioxide concentrations were, respectively, 60 and $19 \mathrm{mg} / \mathrm{L}$. Before yeast inoculation, the must was sterilized by filtration $(0.45 \mu \mathrm{m}$ nitrate cellulose membrane, Millipore, Molsheim, France).

\section{Fermentation Conditions}

Fermentation kinetics were monitored by $\mathrm{CO}_{2}$ release (Bely et al., $1990 \mathrm{a}, \mathrm{b})$. The amount of $\mathrm{CO}_{2}$ release $(\mathrm{g} / \mathrm{L})$ was determined by automatic measurement of fermentor weight loss every $20 \mathrm{~min}$. The $\mathrm{CO}_{2}$ production rate $(\mathrm{g} / \mathrm{L} / \mathrm{h})$ was obtained by polynomial smoothing of the last $11 \mathrm{CO}_{2}$ measurements. The large number of $\mathrm{CO}_{2}$ acquisitions combined with precision weighing $(0.01 \mathrm{~g})$ gave three kinetic parameters with good accuracy: (1) lag phase (h) was the time between inoculation and the beginning of $\mathrm{CO}_{2}$ release, (2) $V \max (\mathrm{g} / \mathrm{L} / \mathrm{h})$ was the maximum $\mathrm{CO}_{2}$ production rate, and (3) FD (h) was the time required to ferment all the sugars in the medium. Weight loss due to evaporation was under $2 \%$.

Yeasts were pre-cultured in Erlenmeyer flasks filled with must at $24^{\circ} \mathrm{C}$ for $24 \mathrm{~h}$ (S. cerevisiae) or $48 \mathrm{~h}$ (T. delbrueckii). Fermentations were carried out at $24^{\circ} \mathrm{C}$ with agitation in 1.2 $\mathrm{L}$ fermenters locked to maintain anaerobiosis throughout $\mathrm{AF}$ ( $\mathrm{CO}_{2}$ was released through a sterile air outlet condenser). Four different trials were carried out: two pure cultures and two mixed cultures. Two types of mixed cultures were carried out: simultaneous mixed culture (called "simultaneous culture") where T. delbrueckii and S. cerevisiae were inoculated at the same time and sequential mixed culture (called "sequential culture") where $T$. delbrueckii was inoculated $24 \mathrm{~h}$ before $S$. cerevisiae yeast. Single and mixed cultures were inoculated with $1 \times 10^{7}$ viable cells $/ \mathrm{mL}$ for $T$. delbrueckii and $2 \times 10^{6}$ viable cells $/ \mathrm{mL}$ for S. cerevisiae. All experiments were performed in triplicate.

\section{Population Kinetics}

In mixed cultures, yeast growth was determined by plate counting on two different agar media. Samples were withdrawn throughout fermentation and diluted appropriately. Non-Saccharomyces cells were counted using a specific agar medium (NS): YPDA (1\% yeast extract, $1 \%$ peptone, $2 \%$ dextrose, $2 \%$ agar; $\mathrm{pH} 4.8$ ) supplemented with $1 \mu \mathrm{g} / \mathrm{mL}$ cycloheximide to promote the growth of T. delbrueckii and inhibit that of S. cerevisiae. This low concentration allowed the growth of T. delbrueckii Zymaflore ${ }^{\circledR}$ Alpha ${ }^{\text {TDn.sacch }}$ but inhibited that of S. cerevisiae Zymaflore ${ }^{\circledR}$ X5 (data not shown). The number of $S$. cerevisiae was given as the difference between the total plate count using YPDA medium and the plate count using NS medium. Yeast growth in single cultures was determined using only the YPDA medium. At the end of AF, we controlled the species by PCR RFLP analysis of rDNA ITS region with digestion by Eco R1 (Granchi et al., 1999). Plates were incubated at $24^{\circ} \mathrm{C}$ for 4 days before counting.

\section{Wine Analysis}

Ethanol concentration (\% vol) was measured by infrared refractance (Spectra Analyser, Axflow, Plaisir, France) and sugar $(\mathrm{g} / \mathrm{L})$ was determined chemically by colorimetry $(460 \mathrm{~nm})$ in continuous flux (Sanimat, Montauban, France). These analyses were performed by Sarco laboratory (Bordeaux, France).

\section{Volatile Thiols Analysis}

Volatile thiol quantification was performed by the wine analysis laboratory Sarco (Bordeaux, France). 4MSP, 3SH, and 3SHA were specifically extracted by reversible combination of the thiols with sodium- $p$-hydroxymercuribenzoate $(p$-HMB), from $50 \mathrm{~mL}$ wine previously preserved from oxidation by adding $50 \mathrm{mg} / \mathrm{L}$ of $\mathrm{SO}_{2}$, as described by Tominaga and Dubourdieu (2006) and quantified by gas chromatography-mass spectrometry according to methods described by Tominaga et al. (1998a) and Tominaga and Dubourdieu (2000).

\section{SH Thiol Precursors Analysis}

Cys-3SH and Glut-3SH were assayed according to the protocol described by Capone et al. (2010), modified as follows. An aliquot $(25 \mu \mathrm{L})$ of an aqueous solution containing $d_{3}$-Glut3SH (final concentration $50 \mu \mathrm{g} / \mathrm{L}$ ) was added to $1 \mathrm{~mL}$ grape juice. The sample was diluted with $2 \mathrm{~mL}$ water and passed through a $6 \mathrm{~mL}, 500 \mathrm{mg}$ LC-18 cartridge (Supelco), previously conditioned with $4 \mathrm{~mL}$ methanol, followed by $2 \mathrm{~mL}$ methanolwater (50/50) mix, and $3 \mathrm{~mL}$ water. After loading the juice, the sorbent was rinsed with $1 \mathrm{~mL}$ water, dried under air for $1 \mathrm{~min}$, and eluted with $3 \mathrm{~mL}$ methanol solution (70\%). The eluate was collected and dried in a Vacuum System with Vortex Motion (RapidVap, Labconco, US) at $10 \mathrm{mbar}$ and $45^{\circ} \mathrm{C}$. The extract was diluted in formic acid solution $(700 \mu \mathrm{L}, 0.1 \%)$, filtered through a $0.45 \mu \mathrm{m}$ filter for LC-MS analysis. All LC-MS analyses were carried out on an Accela UHPLC (Thermo Fisher Scientific), connected in series to an Exactive (Thermo Fisher Scientific, Bremen, Germany) mass spectrometer, equipped with a heated ESI ion source. The column was a $100 \times 2.1 \mathrm{~mm}$, i.d., $1.7 \mu \mathrm{m}$, Synchronis aQ (Thermo Scientific). The solvents were: $0.1 \%$ aqueous formic acid (solvent $\mathrm{A}$ ) and $0.1 \%$ formic acid in acetonitrile (solvent B), with a flow rate of $300 \mu \mathrm{L} / \mathrm{min}$. The gradient for solvent B was as follows: $0 \mathrm{~min}, 9 \%$; $0.8 \mathrm{~min}, 9 \%$; $5 \mathrm{~min}, 40 \%$; $5.2 \mathrm{~min}, 90 \%$. The column was equilibrated with $9 \% \mathrm{~B}$ for $1 \mathrm{~min}$ prior to injection. A $5 \mu \mathrm{L}$ injection volume was used for each sample. The ion source was operated in the positive ion mode at $3.5 \mathrm{kV}$. Source vaporizer temperature was set at $300^{\circ} \mathrm{C}$, capillary temperature at $350^{\circ} \mathrm{C}$, nitrogen sheath gas at 80 , and the auxiliary and sweep gas at 5 (arbitrary units). A mass range of 100-500 was acquired in full scan MS mode. The resolution setting was $25000(\mathrm{~m} / \Delta \mathrm{m}$, fwhm at $\mathrm{m} / \mathrm{z} 400)$.

\section{Statistical Analysis}

In order to compare modalities, data were analyzed by singlefactor variance (ANOVA, $p<0.05$ ), following verification of variance homogeneity (Levene test, $p>0.05$ ). Thereafter, a multiple comparison test (Duncan) was applied to classify the different culture protocols $(p<0.05)$. All statistics were analyzed using the R program. 


\section{RESULTS AND DISCUSSION}

\section{$\mathrm{CO}_{2}$ Release and Population Kinetics in Pure and Mixed Cultures}

Four different AF were conducted in Sauvignon Blanc grape must: two with pure cultures and two with mixed cultures (inoculated either simultaneously or sequentially). In all trials, the must was inoculated with $1 \times 10^{7}$ viable cells $/ \mathrm{mL}$ for T. delbrueckii and $2 \times 10^{6}$ viable cells/mL for $S$. cerevisiae. The final ethanol concentrations ( $12 \%$ vol, corresponding to a final $\mathrm{CO}_{2}$ release of $97 \mathrm{~g} / \mathrm{L}$ ) were reached in all fermentations except in the pure $T$. delbrueckii culture, which predictably stopped fermenting at $6.2 \%$ vol.

The overall fermentation kinetic profiles, i.e., the variation in $\mathrm{CO}_{2}$ rate versus time, are shown in Figure 1. The rate curves varied markedly from one culture to another. Indeed, the trial involving inoculation with $T$. delbrueckii alone showed a short lag phase $(17 \mathrm{~h})$, but also a low fermentation rate, characterized by the lowest $V \max (0.39 \mathrm{~g} / \mathrm{L} / \mathrm{h})$. In contrast, even with a long lag phase $(34 \mathrm{~h})$, the $S$. cerevisiae culture had a high fermentation rate with the highest $V \max (1 \mathrm{~g} / \mathrm{L} / \mathrm{h})$ and the shortest fermentation duration (334 h). These results are in good agreement with previous investigations using a large number of strains (Renault et al., 2009), where T. delbrueckii was found to have a lower fermentation capacity than S. cerevisiae.

Mixed cultures exhibited intermediate fermentation kinetics (Figure 1). When both species were added at the same time, the fermentation curve showed similar profiles to that of pure $S$. cerevisiae culture, but with a lower $V \max (0.84 \mathrm{~g} / \mathrm{L} / \mathrm{h})$ and a shorter lag phase time $(11 \mathrm{~h})$, due to the larger amount of cells inoculated $\left(1.2 \times 10^{7}\right.$ viable cells $\left./ \mathrm{mL}\right)$. On the contrary, when T. delbrueckii and S. cerevisiae were inoculated sequentially, the fermentation curve was close to that of T. delbrueckii alone, except that the $V \max$ was higher $(0.56 \mathrm{~g} / \mathrm{L} / \mathrm{h})$. The fermentation of the sequential culture took longer than that of the simultaneous or pure $S$. cerevisiae cultures.

The viable $S$. cerevisiae and T. delbrueckii populations in pure and mixed cultures were determined by plate counting (Table 1). The biomass kinetics are presented according to AF progress (expressed in $\%$ of $\mathrm{CO}_{2}$ released; Figure 2). The maximum population $(X \max )$ reached during $\mathrm{AF}$ by $T$. delbrueckii and $S$. cerevisiae was higher when they were inoculated alone $\left(8.1 \times 10^{7}\right.$ viable cells $/ \mathrm{mL}$ for $T$. delbrueckii and $7.6 \times 10^{7}$ viable cells $/ \mathrm{mL}$ for $S$. cerevisiae, in pure cultures, respectively) than in sequential and simultaneous cultures. Hence, both species influenced each other's development. It is noteworthy that the $X \max$ of $T$. delbrueckii in the sequential culture was higher $\left(6.1 \times 10^{7}\right.$ viable cells $\left./ \mathrm{mL}\right)$ than in the simultaneous culture $\left(4.3 \times 10^{7}\right.$ viable cells $\left./ \mathrm{mL}\right)$. On the contrary, the $X \max$ of S. cerevisiae was $4.4 \times 10^{7}$ and $2.4 \times 10^{7}$ viable cells $/ \mathrm{mL}$, in simultaneous and sequential cultures, respectively.

Indeed, in sequential culture, when the addition of $S$. cerevisiae (when $2.5 \mathrm{~g} / \mathrm{L} \mathrm{CO}_{2}$ had been released) was delayed, T. delbrueckii was able to grow from $1 \times 10^{7}$ viable cells $/ \mathrm{mL}$ to $4.1 \times 10^{7}$ viable cells/mL within the first $24 \mathrm{~h}$, thus initiating AF (Figure 2). In that case, the $T$. delbrueckii/S. cerevisiae ratio after $24 \mathrm{~h}$ was largely in favor of $T$. delbrueckii (about 20:1) but $S$. cerevisiae developed sufficiently (from $2 \times 10^{6}$ to $2.4 \times 10^{7}$ viable cells $/ \mathrm{mL}$ ) to complete AF.

Consequently, in the sequential culture, the $X \max$ of T. delbrueckii was maintained during the first $60 \%$ of $\mathrm{AF}$ and its viable population was higher than that of $S$. cerevisiae during the first $85 \%$ of AF (Figure 2). The dominance of $T$. delbrueckii throughout the $\mathrm{AF}$, in sequential culture, is probably due to higher consumption of dissolved oxygen, nitrogen and vitamins than S. cerevisiae which was inoculated $24 \mathrm{~h}$ after.

The kinetics of the two yeast populations were very different following simultaneous inoculation, where the initial inoculation ratio of 5:1 $\left(1 \times 10^{7}\right.$ viable cells/mL T. delbrueckii and $2 \times 10^{6}$

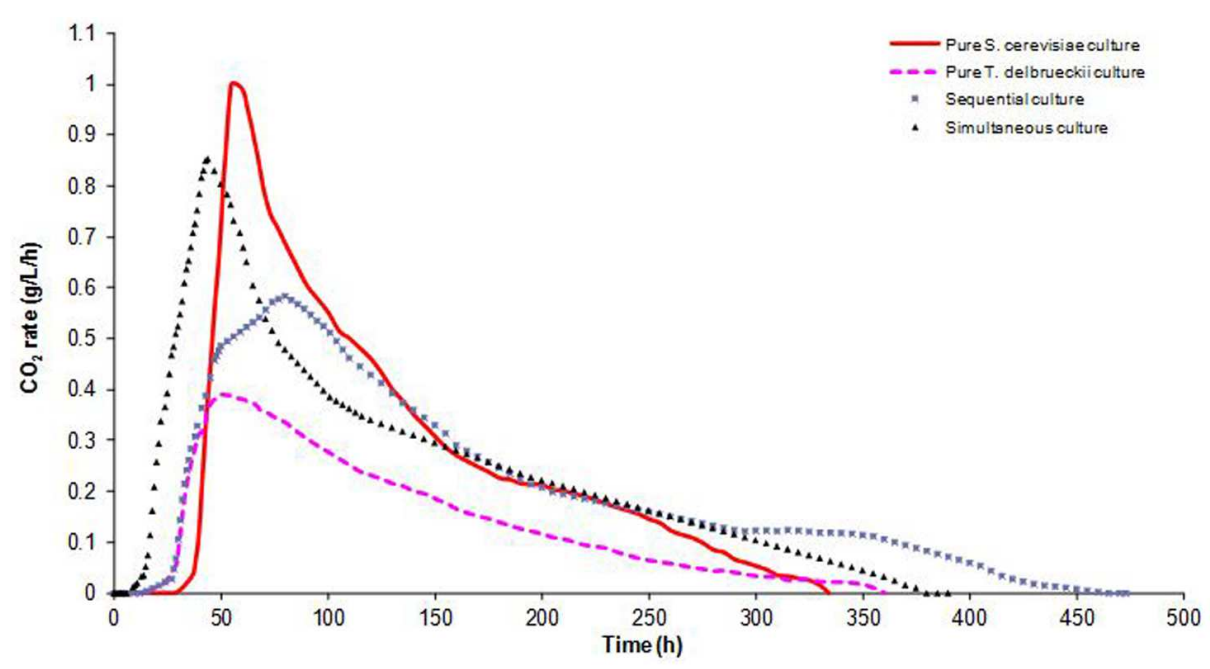

FIGURE $1 \mid \mathrm{CO}_{2}$ production rates (g/L/h) over time in pure and mixed $\mathbf{T}$. delbrueckii/S. cerevisiae cultures. Average values of three experiments, standard deviation $<5 \%$. 
TABLE 1 | Maximal cell population and final volatile thiol concentrations in pure and mixed T. delbrueckii and S. cerevisiae cultures.

\begin{tabular}{|c|c|c|c|c|}
\hline & $\begin{array}{l}\text { Torulaspora delbrueckii } \\
\text { pure culture }\end{array}$ & $\begin{array}{l}\text { Sequential } \\
\text { mixed culture }\end{array}$ & $\begin{array}{l}\text { Simultaneous } \\
\text { mixed culture }\end{array}$ & $\begin{array}{c}\text { Saccharomyces cerevisiae } \\
\text { pure culture }\end{array}$ \\
\hline \multicolumn{5}{|c|}{ Maximal population (viable cells $/ \mathrm{mL}$ ) } \\
\hline S. cerevisiae & / & $2.4 \times 10^{7} \pm 7.1 \times 10^{5 a}$ & $4.4 \times 10^{7} \pm 2.3 \times 10^{6 b}$ & $7.6 \times 10^{7} \pm 1.8 \times 10^{6 \mathrm{c}}$ \\
\hline \multicolumn{5}{|c|}{ Final volatile thiol concentrations (ng/L) } \\
\hline $3 \mathrm{SH}$ & $623 \pm 103^{b}$ & $1312 \pm 224^{c}$ & $362 \pm 97^{\mathrm{a}}$ & $303 \pm 141^{\mathrm{a}}$ \\
\hline
\end{tabular}

Average values of three experiments \pm standard deviation. ${ }^{a, b, c}$ Represents significantly different statistical groups $(p<0.05)$. 3SH, 3-sulfanylhexan-1-ol; 3SHA, 3sulfanylhexyl acetate.
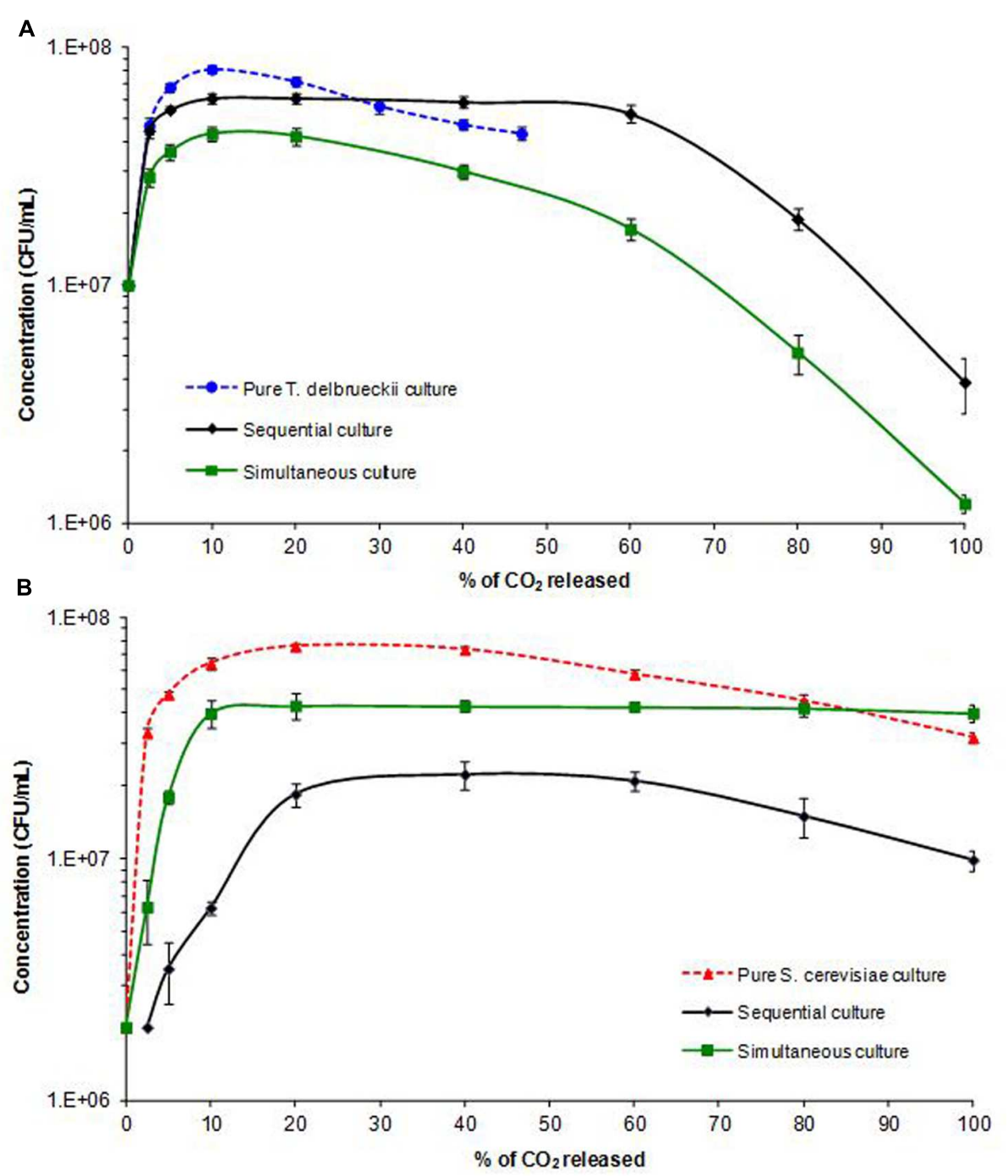

FIGURE 2 | Kinetics of T. delbrueckii (A) and S. cerevisiae (B) cell populations (CFU/mL) during alcoholic fermentation (AF) in pure and mixed cultures. Average value of three experiments.

viable cells/mL S. cerevisiae) was less favorable to T. delbrueckii, which was only dominant during the first $10 \%$ of AF. Indeed, the $X \max$ of $T$. delbrueckii in simultaneous inoculation was lower than in sequential culture $\left(4.3 \times 10^{7}\right.$ and $6.1 \times 10^{7}$ viable
cells/mL, respectively). Furthermore, S. cerevisiae also reached its $X$ max during the early stage of AF and maintained this level of population until the end of AF, whereas the viable T. delbrueckii population decreased rapidly after $10 \%$ of AF. According to 
several authors (Nissen and Arneborg, 2003; Nissen et al., 2003; Renault et al., 2013), the physical contact/proximity between $T$. delbrueckii and a large viable population of $S$. cerevisiae induced the rapid death of $T$. delbrueckii. Competition for oxygen may also explain the rapid death of $T$. delbrueckii cells (Hansen et al., 2001). Indeed, while S. cerevisiae yeast is able to grow rapidly under strictly anaerobic conditions, $T$. delbrueckii is affected by a lack of oxygen (Hanl et al., 2005).

To sum up, sequential culture facilitated the development of $T$. delbrueckii, resulting in a larger viable population than that of S. cerevisiae almost until the end of AF. Under these conditions, the kinetic parameters were close to those obtained in pure $T$. delbrueckii culture, except that $\mathrm{AF}$ was completed. In contrast, when both species were inoculated simultaneously, the maximal viable populations of both species were similar, but that of S. cerevisiae was larger than that of $T$. delbrueckii during $90 \%$ of the reaction, with AF showing similar profiles to those of pure S. cerevisiae cultures. Nevertheless, T. delbrueckii had a small impact on fermentation kinetics, as $V \max$ was lower and AF was extended, in comparison to the pure S. cerevisiae culture.

\section{Volatile Thiol Production}

Torulaspora delbrueckii in pure culture did not produce 4MSP, unlike S. cerevisiae $(33 \mathrm{ng} / \mathrm{L}$ at the end of AF). Very small amounts were detected in mixed cultures $(<7 \mathrm{ng} / \mathrm{L})$, suggesting the absence of any interaction between the species in producing this compound. These results confirmed the inability of $T$. delbrueckii to release 4MSP, as already reported in previous studies (Zott et al., 2011; Sadoudi et al., 2012).

As shown Figure 3, 3SH production was similar in both pure cultures during the first $20 \%$ of AF but diverged after this point, with differences in the final concentrations. Indeed, at the end of fermentation, the $3 \mathrm{SH}$ concentration in the pure T. delbrueckii culture was twofold higher than that in the pure S. cerevisiae culture (623 and $303 \mathrm{ng} / \mathrm{L}$, respectively; Table 1). This result differed from previous findings using other T. delbrueckii strains (Zott et al., 2011; Sadoudi et al., 2012), suggesting that this production is strain-dependent.

Results were different for 3SHA for which T. delbrueckii produced very small amounts $(14 \mathrm{ng} / \mathrm{L})$, showing a progressive and linear production throughout AF (Figure 3). On the contrary, total 3SHA production by $S$. cerevisiae was higher $(83 \mu \mathrm{g} / \mathrm{L})$, with $50 \%$ occurring during the growth phase. Furthermore, the $3 \mathrm{SH} / 3 \mathrm{SHA}$ ratios were 3.6 and 44.5 in pure S. cerevisiae and T. delbrueckii cultures, respectively. These results confirmed those obtained by Sadoudi et al. (2012), showing that $T$. delbrueckii had a lower acetylation activity (i.e., a low conversion rate of $3 \mathrm{SH}$ to $3 \mathrm{SHA}$ ) than S. cerevisiae (Coetzee and du Toit, 2012).

Furthermore, no significant difference in $3 \mathrm{SH}$ and $3 \mathrm{SHA}$ production was observed between the simultaneous and pure S. cerevisiae cultures at the end of AF (Table 1). However, it is interesting to note that, in the simultaneous inoculation protocol, the beginning of production was delayed (no production during the first 10\% of AF; Figure 3).

Concerning the sequentially inoculated culture, $3 \mathrm{SH}$ production was similar to that of the pure S. cerevisiae culture until $20 \%$ of AF, but diverged beyond that point, exhibiting a major increase during the last stage in $\mathrm{AF}$, resulting in significantly different final concentrations. Indeed, at the end of $\mathrm{AF}$, the $3 \mathrm{SH}$ concentration in the sequential culture was fourfold higher than in the pure S. cerevisiae culture (1312 and $303 \mathrm{ng} / \mathrm{L}$, respectively; Table 1). 3SHA production in sequential culture was also different from the pure $S$. cerevisiae culture, remaining very weak until $20 \%$ of $\mathrm{AF}$ and then drastically increasing to reach a final concentration nearly threefold higher than in the pure S. cerevisiae culture (218 and $83 \mathrm{ng} / \mathrm{L}$, respectively; Figure 3).

These results suggested that sequential inoculation of $S$. cerevisiae and $T$. delbrueckii in Sauvignon Blanc must resulted in synergistic interactions that affected 3SH and 3SHA production during AF.

\section{Volatile Thiol Precursors}

To investigate the possible synergistic interactions between the two species resulting in higher concentrations of $3 \mathrm{SH}$ and its acetate at the end of AF, their $S$-conjugate precursors (Cys$3 \mathrm{SH}$ and Glut-3SH) were monitored throughout fermentation (Figure 4). Cys-3SH and Glut-3SH were detected ( $0 \%$ of $\mathrm{AF}$ ) at normal levels for a Sauvignon Blanc must: $20 \mu \mathrm{g} / \mathrm{L}$ and $160 \mu \mathrm{g} / \mathrm{L}$, respectively.

In pure S. cerevisiae and T. delbrueckii cultures, only $60 \%$ of the initial Glut-3SH concentration was assimilated, leaving approximately $65 \mu \mathrm{g} / \mathrm{L}$ of the precursor in the wine. However, in simultaneous and sequential cultures uptake was significantly higher. Indeed, up to 69 and 79\% of Glut-3SH were assimilated, resulting in wines with 50 and $33 \mu \mathrm{g} / \mathrm{L}$ precursor, respectively.

Figure 4 shows that, for all modalities, Glut-3SH was rapidly metabolized by the yeast in the earliest stage of AF, immediately after yeast addition. In the pure $S$. cerevisiae culture, the uptake stopped at $20 \%$ of AF. In all cultures involving T. delbrueckii, Glut-3SH kinetics were similar until $5 \%$ of $\mathrm{AF}$, then the assimilation slowed down suddenly and stopped around $20 \%$ of AF in pure T. delbrueckii and simultaneous cultures. In the sequential culture, Glut-3SH assimilation continued slowly after $20 \%$ of AF, resulting in the lowest final Glut-3SH concentration in the medium. This enhanced precursor assimilation may explain the variations in total $3 \mathrm{SH}$ and $3 \mathrm{SHA}$ release observed at end of AF.

The kinetics of cysteine S-conjugate precursor (Cys-3SH) concentrations during AF is probably due to the fact that it is both produced and assimilated by the yeast (Cordente et al., 2015). Cys-3SH kinetics varied markedly from one culture to another (Figure 4). In the pure S. cerevisiae culture, Cys$3 \mathrm{SH}$ concentrations decreased after a short lag phase $(<5 \%$ of $\mathrm{AF}$ ) and completely disappeared after $20 \%$ of AF. In contrast, in all cultures involving $T$. delbrueckii, an increase in Cys$3 \mathrm{SH}$ concentrations was observed in the early stage of $\mathrm{AF}$ (Figure 4). In the pure T. delbrueckii culture, the concentration increased by $50 \%(21 \mu \mathrm{g} / \mathrm{L}$ initial to $32 \mu \mathrm{g} / \mathrm{L}$ at $5 \%$ of $\mathrm{AF})$ during the very early stages of AF. In this phase, Cys-3SH production was concomitant to Glut-3SH depletion (Figure 4) and $3 \mathrm{SH}$ release (Figure 3), suggesting that T. delbrueckii was able to synthesize Cys-3SH and $3 \mathrm{SH}$ from Glut-3SH, but the 

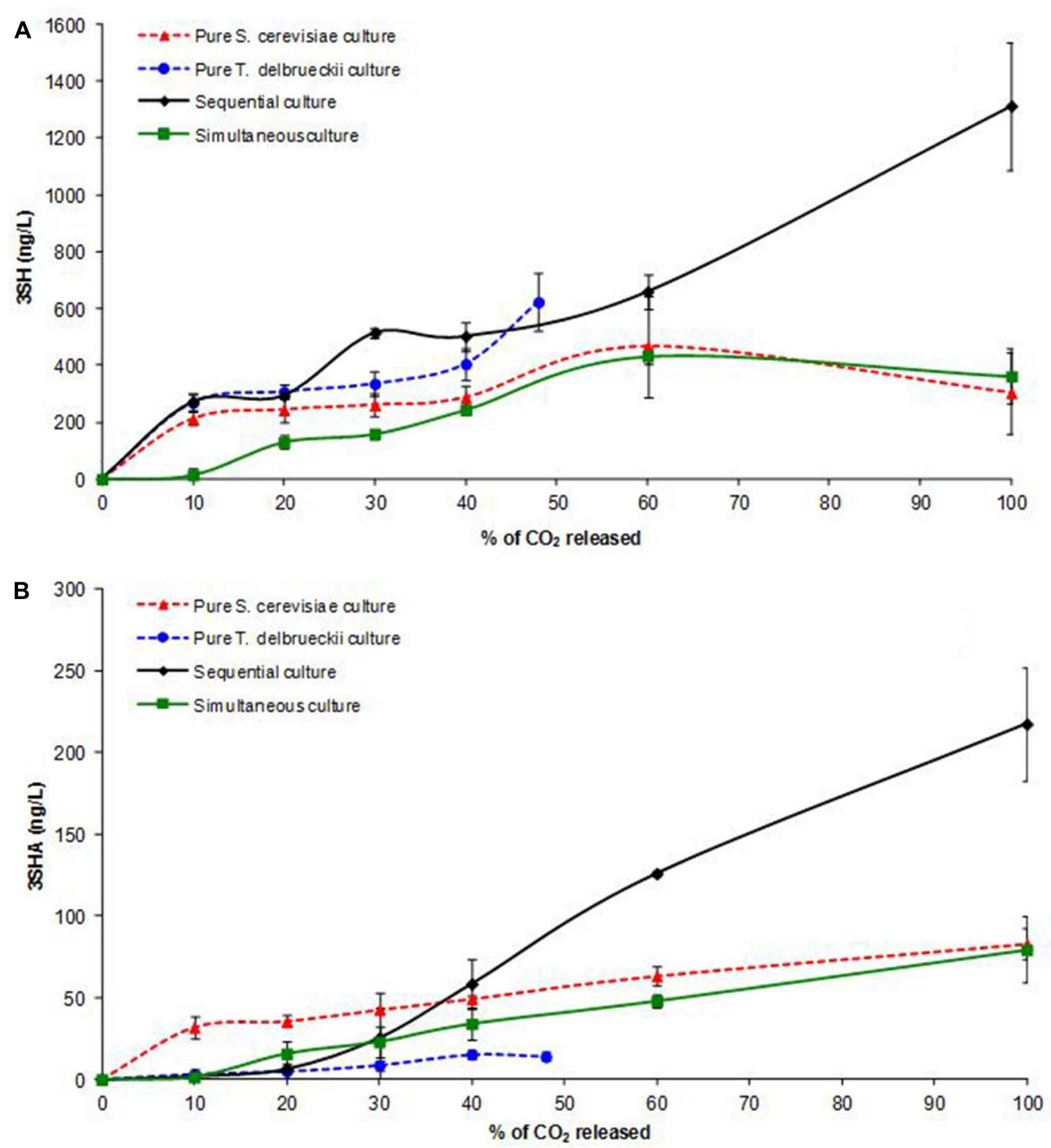

FIGURE 3 | Kinetics of 3SH (A) and 3SHA (B) concentrations (ng/L) in pure and mixed T. delbrueckii/S. cerevisiae cultures during AF. Average value of three experiments.

cysteinylated form was hardly assimilated, if at all, by the yeast. In $S$. cerevisiae, its transport is provided by the Gap $1 p$ membrane protein (Subileau et al., 2008a). The GAP1 gene has not been clearly identified in the genome sequence of T. delbrueckii, type strain CBS $1146 \mathrm{~T}$ (CLIB230T). Indeed, the closest BLAST of Gap1p protein from S. cerevisiae S288c against CBS $1146 \mathrm{~T}$ is a hypothetical protein (TDEL_0C00930) with only $74 \%$ identity along $96 \%$ of the sequence. It was, therefore, hypothesized that Gaplp permease was absent or dysfunctional in this species. Further experiments are required to validate this hypothesis, for example, an intracellular Cys-3SH assay.

When Glut-3SH stopped being converted into Cys-3SH, its concentration in the medium remained constant until the end of AF.

Interestingly, throughout $\mathrm{AF}$ in the two mixed cultures, the more T. delbrueckii developed, the higher the Cys-3SH concentration became. In the sequential culture, where
T. delbrueckii dominated S. cerevisiae for $85 \%$ of AF (Figure 2), the Cys-3SH accumulation phase in the must was much longer than in the simultaneous culture, resulting in a higher concentration at the end of this phase $(34 \mu \mathrm{g} / \mathrm{L}$ instead of $27 \mu \mathrm{g} / \mathrm{L})$. After this accumulation phase, in both mixed cultures, an abrupt depletion reduced Cys-3SH to undetectable levels in the medium after around 40 and $60 \%$ of $\mathrm{AF}$, in simultaneous and sequential cultures, respectively. It is worth noting that Cys-3SH uptake was apparently correlated with the development of $S$. cerevisiae (Figure 2). Precursor uptake began when $S$. cerevisiae reached its $X \max$, around 10 and $20 \%$ of $\mathrm{AF}$ (corresponding to $4.3 \times 10^{7}$ and $2.4 \times 10^{7}$ viable cells $/ \mathrm{mL}$ in simultaneous and sequential cultures, respectively). Moreover, the Cys-3SH degradation rate (i.e., the slope of the line) of both mixed culture was apparently correlated to S. cerevisiae Xmax level. The steep slope observed for the pure $S$ cerevisiae culture, with $X \max$ around $7.5 \times 10^{7}$ viable cells $/ \mathrm{mL}$, supports this hypothesis. 

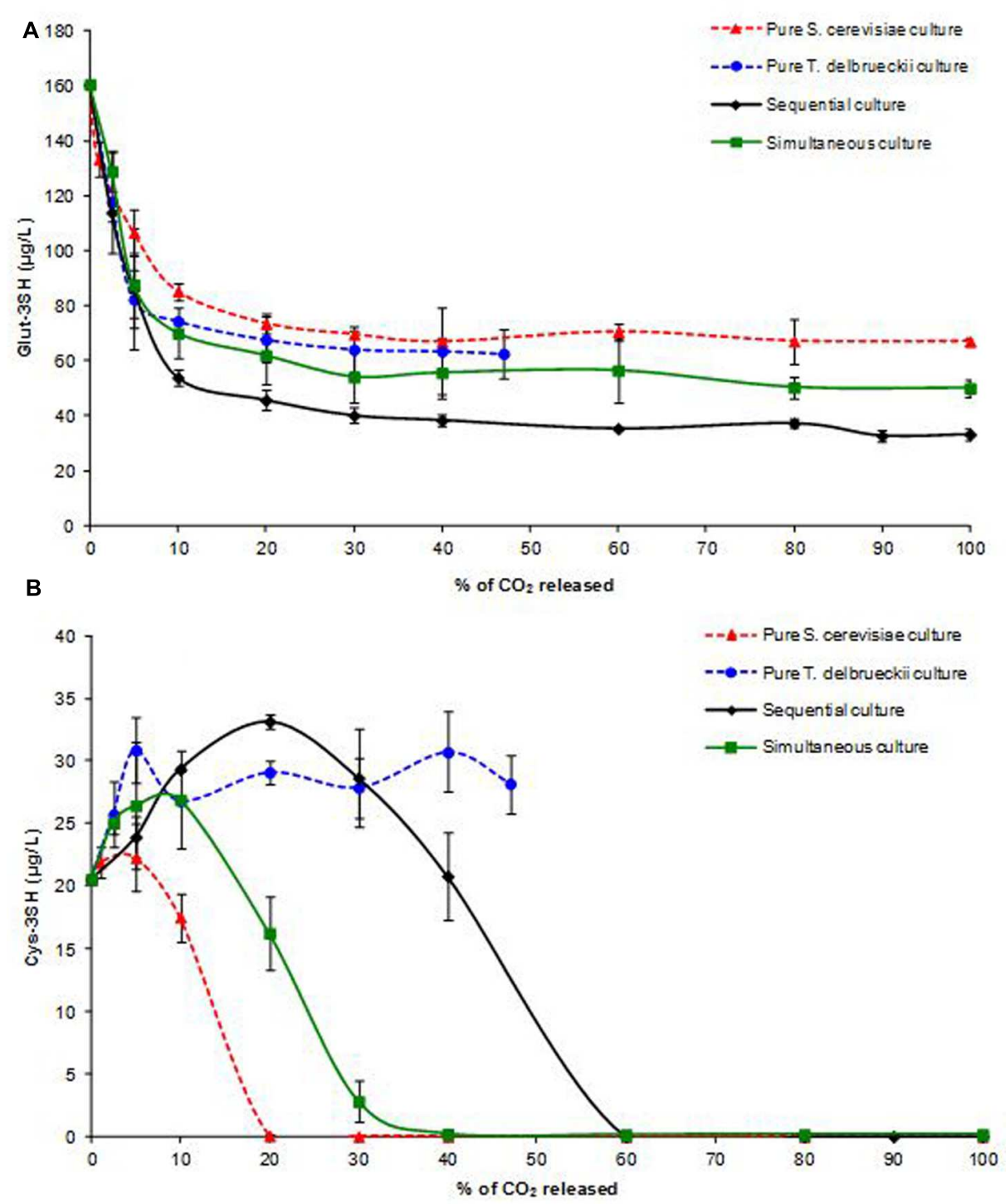

FIGURE 4 | Kinetics of Glut-3SH (A) and Cys-3SH (B) concentrations ( $\mu \mathrm{g} / \mathrm{L})$ in pure and mixed T. delbrueckii/S. cerevisiae cultures during AF. Average value of three experiments.

\section{CONCLUSION}

This study, based on an analysis of the main thiols throughout AF of Sauvignon Blanc must fermented by $T$. delbrueckii and $S$. cerevisiae in pure or mixed cultures, provides interesting insights into the metabolic pathway of thiols in T. delbrueckii and reveals a synergistic interaction between the two species.

Under these experimental conditions, T. delbrueckii produced no 4MSP and only very small amounts of 3SHA, confirming previous findings. In contrast, high $3 \mathrm{SH}$ levels were found in wines fermented with pure $T$. delbrueckii and sequential $T$. delbrueckii/S. cerevisiae cultures, in comparison to wines resulting from AF with only $S$. cerevisiae. Monitoring $3 \mathrm{SH}$ and its precursors (Glut-3SH and Cys-3SH) throughout $\mathrm{AF}$ led us to conclude that $T$. delbrueckii only assimilates the glutathionylated precursor, while both precursor forms are metabolized by $S$. cerevisiae. In pure $T$. delbrueckii cultures, Glut-3SH degradation produced significant amounts of $3 \mathrm{SH}$ and Cys-3SH in the wine. In mixed cultures, the more T. delbrueckii developed, the higher the Glut-3SH uptake and Cys-3SH release. In sequential cultures, which favored T. delbrueckii development compared to the simultaneous protocol, the results revealed an increase in the cysteinylated precursor followed by an increase in $3 \mathrm{SH}$. Hence, once released by T. delbrueckii, the cysteinylated precursor was converted into $3 \mathrm{SH}$ by S. cerevisiae in the last stage of AF. The direct consequence was higher overall $3 \mathrm{SH}$ and 3SHA production than in pure S. cerevisiae cultures. Further work with different strains of $T$. delbrueckii and S. cerevisiae will help to confirm the synergistic interaction described between these two species. 


\section{AUTHOR CONTRIBUTIONS}

Conceived and designed the experiments: JC, VM, CT, MB, and PR. Performed the experiments: PR and CT.

\section{REFERENCES}

Azzolini, M., Fedrizzi, B., Tosi, E., and Finato, F. (2012). Effects of Torulaspora delbrueckii and Saccharomyces cerevisiae mixed cultures on fermentation and aroma of Amarone wine. Food Res. Technol. 235, 303-313. doi: 10.1007/s00217012-1762-3

Bely, M., Sablayrolles, J. M., and Barre, P. (1990a). Automatic detection of assimilable nitrogen deficiencies during alcoholic fermentation in oenological conditions. J. Ferment. Bioeng. 70, 246-252. doi: 10.1016/0922-338X(90) 90057-4

Bely, M., Sablayrolles, J. M., and Barre, P. (1990b). Description of alcoholic fermentation kinetics: its variability and significance. Am. J. Enol. Vitic. 41, 319-324.

Cabrera, M. J., Moreno, J., Ortega, J. M., and Medina, M. (1988). Formation of ethanol, higher alcohols, esters, and terpenes by five yeast strains in musts from Pedro Ximenez grapes in various degrees of ripeness. Am. J. Enol. Vitic. 39, $283-287$.

Capone, D. L., Sefton, M. A., Hayasaka, Y., and Jeffery, D. W. (2010). Analysis of precursors to wine odorant 3-mercaptohexan-1-ol using HPLCMS/MS: resolution and quantitation of diastereomers of 3-S-cysteinylhexan1-ol and 3-S-glutathionylhexan-1-ol. J. Agric. Food Chem. 58, 1390-1395. doi: $10.1021 /$ jf903720w

Ciani, M., and Maccarelli, F. (1998). Oenological properties of non-Saccharomyces yeasts associated with wine-making. World J. Microbiol. Biotechnol. 14, 199-203. doi: 10.1023/A:1008825928354

Ciani, M., and Picciotti, G. (1995). The growth-kinetics and fermentation behavior of some non-Saccharomyces yeasts associated with winemaking. Biotechnol. Lett. 17, 1247-1250. doi: 10.1007/BF001 28395

Coetzee, C., and du Toit, W. J. (2012). A comprehensive review on Sauvignon blanc aroma with a focus on certain positive volatile thiols. Food Res. Int. 45, 287-298. doi: 10.1016/j.foodres.2011.09.017

Comitini, F., Gobbi, M., Domizio, P., Romani, C., Lencioni, L., Mannazzu, I., et al. (2011). Selected non-Saccharomyces wine yeasts in controlled multistarter fermentations with Saccharomyces cerevisiae. Food Microbiol. 28, 873-882. doi: 10.1016/j.fm.2010.12.001

Cordente, A. G., Capone, D. L., and Curtin, C. D. (2015). Unravelling glutathione conjugate catabolism in Saccharomyces cerevisiae: the role of glutathione/dipeptide transporters and vacuaolar function in the release of volatile sulfur compounds 3-mercaptohexan-1-ol and 4-mercapto4methylpentan-2-one. Appl. Microbiol. Biotechnol. 99, 9709-9722. doi: 10.1007/s00253-015-6833-5

Darriet, P., Tominaga, T., Lavigne, V., Boidron, J. N., and Dubourdieu, D. (1995). Identification of a powerful aromatic component of Vitis vinifera L. var. Sauvignon wines: 4-mercapto-4-methylpentan-2-one. Flavour Frag. J. 10, 385-392. doi: 10.1002/ffj.2730100610

Dubourdieu, D., Tominaga, T., Masneuf, I., Gachons, C., and Murat, M. L. (2006). The role of yeasts in grape flavor development during fermentation: the example of Sauvignon blanc. Am. J. Enol. Vitic. 57, 81-88.

Fedrizzi, B., Pardon, K. H., Sefton, M. A., Elsey, G. M., and Jeffery, D. W. (2009). First Identification of 4-S-Glutathionyl-4-methylpentan-2-one, a Potential Precursor of 4-Mercapto-4-methylpentan-2-one, in Sauvignon Blanc Juice. J. Agric. Food Chem. 57, 991-995. doi: 10.1021/jf802799w

Granchi, L., Bosco, M., Messini, A., and Vincenzini, M. (1999). Rapid detection and quantification of yeast species during spontaneous wine fermentation by PCR-RFLP analysis of the rDNA ITS region. J. Appl. Microbiol. 87, 949-956. doi: 10.1046/j.1365-2672.1999.00600.x

Grant-Preece, P. A., Pardon, K. H., Capone, D. L., Cordente, A. G., Sefton, M. A., Jeffery, D. W., et al. (2010). Synthesis of wine thiol conjugates and labeled analogues: fermentation of the glutathione conjugate of 3-mercaptohexan-1-ol

\section{ACKNOWLEDGMENTS}

The authors thank Inno'vin for its precious help and Aquitaine Regional Council for its financial support.

yields the corresponding cysteine conjugate and free thiol. J. Agric. Food Chem. 58, 1383-1389. doi: 10.1021/jf9037198

Hanl, L., Sommer, P., and Arneborg, N. (2005). The effect of decreasing oxygen feed rates on growth and metabolism of Torulaspora delbrueckii. Appl. Microbiol. Bioteachnol. 67, 113-118. doi: 10.1007/s00253-004-1695-2

Hansen, E. H., Nissen, P., Sommer, P., Nielsen, J. C., and Arneborg, N. (2001). The effect of oxygen on the survival of non-Saccharomyces yeasts during mixed culture fermentations of grape juice with Saccharomyces cerevisiae. J. Appl. Microbiol. 91, 541-547. doi: 10.1046/j.1365-2672.2001.01426.x

Hernandez-Orte, P., Cersosimo, M., Loscos, N., Cacho, J., Garcia-Moruno, E., and Ferreira, V. (2008). The development of varietal aroma from nonfloral grapes by yeasts of different genera. Food Chem. 107, 1064-1077. doi: 10.1016/j.foodchem.2007.09.032

Herraiz, T., Reglero, G., Herraiz, M., Martin-Alvarez, P. J., and Cabezudo, M. D. (1990). The influence of the yeast and type of culture on the volatile composition of wines fermented without sulfur dioxide. Am. J. Enol. Vitic. 41, 313-318.

Holt, S., Cordente, A. G., Williams, S. J., Capone, D. L., Jitjaroen, W., Menz, I. R., et al. (2011). Engineering Saccharomyces cerevisiae to release 3-mercaptohexan-1-ol during Fermentation through Overexpression of an S. cerevisiae Gene, STR3, for improvement of wine aroma. Appl. Environ. Microbiol. 77, 3626-3632. doi: 10.1128/AEM.03009-10

Howell, K. S., Klein, M., Swiegers, J. H., Hayasaka, Y., Elsey, G. M., Fleet, G. H., et al. (2005). Genetic determinants of volatile-thiol release by Saccharomyces cerevisiae during wine fermentation. Appl. Environ. Microbiol. 71, 5420-5426. doi: 10.1128/AEM.71.9.5420-5426.2005

King, A., and Richard Dickinson, J. (2000). Biotransformation of monoterpene alcohols by Saccharomyces cerevisiae, Torulaspora delbrueckii and Kluyveromyces lactis. Yeast 16, 499-506. doi: 10.1002/(SICI)1097-0061(200004) 16:6<499::AID-YEA548> 3.0.CO;2-E

Kobayashi, H., Takase, H., Kaneko, K., Tanzawa, F., Takata, R., Suzuki, S., et al. (2010). Analysis of S-3-(Hexan-1-ol)-Glutathione and S-3-(Hexan-1-ol)-LCysteine in Vitis vinifera L. cv. Koshu for Aromatic Wines. Am. J. Enol. Vitic. 61, 176-185.

Martinez, J., Toledano, F., Millan, C., and Ortega, J. M. (1990). Development of alcoholic fermentation in non-sterile musts from Pedro Ximenez grapes inoculated with pure cultures of selected yeasts. Food Microbiol. 7, 217-225. doi: 10.1016/0740-0020(90)90027-F

Marullo, P., and Dubourdieu, D. (2010). Yeast selection for wine flavour modulation. Manag. Wine Qual. 2, 293-345. doi: 10.1533/9781845699987.2.293

Murat, M. L., Masneuf, I., Darriet, P., Lavigne, V., Tominaga, T., and Dubourdieu, D. (2001). Effect of Saccharomyces cerevisiae yeast strains on the liberation of volatile thiols in Sauvignon blanc wine. Am. J. Enol. Vitic. 52, 136-139.

Nissen, P., and Arneborg, N. (2003). Characterization of early deaths of nonSaccharomyces yeasts in mixed cultures with Saccharomyces cerevisiae. Arch. Microbiol. 180, 257-263. doi: 10.1007/s00203-003-0585-9

Nissen, P., Nielsen, D., and Arneborg, N. (2003). Viable Saccharomyces cerevisiae cells at high concentrations cause early growth arrest of non-Saccharomyces yeasts in mixed cultures by a cell-cell contact-mediated mechanism. Yeast 20, 331-341. doi: 10.1002/yea.965

Peyrot des Gachons, C., Tominaga, T., and Dubourdieu, D. (2002). Sulfur aroma precursor present in S-glutathione conjugate form: identification of S-3-(hexan-1-ol)-glutathione in must from Vitis vinifera L. cv. Sauvignon blanc. J. Agr. Food Chem. 50, 4076-4079. doi: 10.1021/jf020002y

Plata, C., Millan, C., Mauricio, J. C., and Ortega, J. M. (2003). Formation of ethyl acetate and isoamyl acetate by various species of wine yeasts. Food Microbiol. 20, 217-224. doi: 10.5803/jsfm.20.217

Renault, P., Albertin, W., and Bely, M. (2013). An innovative tool reveals interaction mechanisms among yeast populations under 
oenological conditions. Appl. Microbiol. Biotechnol. 97, 4105-4119. doi: 10.1007/s00253-012-4660-5

Renault, P., Coulon, J., de Revel, G., Barbe, J. C., and Bely, M. (2015). Increase of fruity aroma during mixed T. delbrueckii and $S$. cerevisiae wine fermentation is linked to specific esters enhancement. Int. J. Food Microbiol. 207, 40-48. doi: 10.1016/j.ijfoodmicro.2015.04.037

Renault, P., Miot-Sertier, C., Marullo, P., Hernández-Orte, P., Lagarrigue, L., Lonvaud-Funel, A., et al. (2009). Genetic characterization and phenotypic variability in Torulaspora delbrueckii species: potential applications in the wine industry. Int. J. Food Microbiol. 134, 201-210. doi: 10.1016/j.ijfoodmicro.2009.06.008

Roland, A., Schneider, R., Razungles, A., and Cavelier, F. (2011). Varietal thiols in wine: discovery, analysis and applications. Chem. Rev. 111, 7355-7376. doi: $10.1021 / \mathrm{cr} 100205 \mathrm{~b}$

Roncoroni, M., Santiago, M., Hooks, D. O., Moroney, S., Harsch, M. J., Lee, S. A., et al. (2011). The yeast IRC7 gene encodes a b-lyase responsible for production of the varietal thiol 4-mercapto-4-methylpentan-2-one in wine. Food Microbiol. 28, 926-935. doi: 10.1016/j.fm.2011.01.002

Sadoudi, M., Tourdot-Maréchal, R., Rousseaux, S., Steyer, D., Gallardo-Chacón, J. J., Ballester, J., et al. (2012). Yeast-yeast interactions revealed by aromatic profile analysis of Sauvignon blanc wine fermented by single or co-culture of non-Saccharomyces and Saccharomyces yeasts. Food Microbiol. 32, 243-253. doi: 10.1016/j.fm.2012.06.006

Shinohara, T., Kubodera, S., and Yanagida, F. (2000). Distribution of phenolic yeasts and production of phenolic off-flavors in wine fermentation. J. Biosci. Bioeng. 90, 90-97. doi: 10.1016/S1389-1723(00)80040-7

Subileau, M., Schneider, R., Salmon, J. M., and Degryse, E. (2008a). New insights on 3-mercaptohexanol (3MH) biogenesis in Sauvignon Blanc wines: Cys-3MH and (E)-hexen-2-al are not the major precursors. J. Agr. Food Chem. 56, 9230-9235. doi: 10.1021/jf801626f

Subileau, M., Schneider, R., Salmon, J. M., and Degryse, E. (2008b). Nitrogen catabolite repression modulates the production of aromatic thiols characteristic of Sauvignon Blanc at the level of precursor transport. FEMS Yeast Res. 8, 771-780. doi: 10.1111/j.1567-1364.2008.00400.x

Swiegers, J. H., Willmott, R., Hill-Ling, A., Capone, D. L., Pardon, K. H., Elsey, G. M., et al. (2006). Modulation of volatile thiol and ester aromas by modified wine yeast. Dev. Food Sci. 43, 113-116. doi: 10.1016/S0167-4501(06)80027-0

Thibon, C., Marullo, P., Claisse, O., Cullin, C., Dubourdieu, D., and Tominaga, T. (2008). Nitrogen catabolic repression controls Saccharomyces cerevisiae. FEMS Yeast Res. 8, 1076-1086. doi: 10.1111/j.1567-1364.2008.00381.x

Tominaga, T. (1998). Recherches Sur l'arôme Variétal des Vins de Vitis vinifera L. cv. Sauvignon blanc et sa Genèse à Partir de Précurseurs Inodores du Raisin, Thèse de doctorat, Université de Bordeaux 2, Bordeaux.

Tominaga, T., Darriet, P., and Dubourdieu, D. (1996). Identification of 3mercaptohexyl acetate in Sauvignon wine, a powerful aromatic compound exhibiting box-tree odor. Vitis 35, 207-210.
Tominaga, T., and Dubourdieu, D. (1997). Identification of 4-mercapto-4methylpentan-2-one from the box tree (Buxus sempervirens L.) and broom (Sarothamnus scoparius (L.) Koch.). Flavour Frag. J. 12, 373-376. doi: 10.1002/(SICI)1099-1026(199711/12)12:6<373::AID-FFJ675>3.0.CO;2-Y

Tominaga, T., and Dubourdieu, D. (2000). Identification of cysteinylated aroma precursors of certain volatile thiols in passion fruit juice. J. Agric. Food Chem. 48, 2874-2876. doi: 10.1021/jf990980a

Tominaga, T., and Dubourdieu, D. (2006). A novel method for quantification of 2-methyl-3-furanthiol and 2-furanmethanethiol in wines made from Vitis vinifera grape varieties. J. Agric. Food Chem. 54, 29-33. doi: 10.1021/jf05 0970b

Tominaga, T., Furrer, A., Henry, R., and Dubourdieu, D. (1998a). Identification of new volatile thiols in the aroma of Vitis vinifera L. var. Sauvignon blanc wines. Flavour Frag. J. 13, 159-162. doi: 10.1002/(SICI)1099-1026(199805/06) $13: 3<159::$ AID-FFJ709>3.0.CO;2-7

Tominaga, T., Gachons, C., and Dubourdieu, D. (1998b). A new type of flavor precursors in Vitis vinifera L. cv. Sauvignon blanc: S-cysteine conjugates. J. Agric. Food Chem. 46, 5215-5219. doi: 10.1021/jf980481u

Velázquez, R., Zamora, E., Álvarez, M. L., Hernández, L. M., and Ramírez, M. (2015). Effects of new Torulaspora delbrueckii killer yeasts on the must fermentation kinetics and aroma compounds of white table wine. Front. Microbiol. 6:1222. doi: 10.3389/fmicb.2015.01222

Viana, F., Gil, J. V., Genovés, S., Vallés, S., and Manzanares, P. (2008). Rational selection of non-Sacharomyces wine yeasts for mixed starters based on ester formation and enological traits. Food Microbiol. 25, 778-785. doi: 10.1016/j.fm.2008.04.015

Winter, G., Van Der Westhuizen, T., Higgins, V. J., Curtin, C., and Ugliano, M. (2011). Contribution of cysteine and glutathione conjugates to the formation of the volatile thiols 3-mercaptohexan-1-ol (3MH) and 3-mercaptohexyl acetate (3MHA) during fermentation by Saccharomyces cerevisiae. Aust. J. Grape Wine Res. 17, 285-290. doi: 10.1111/j.1755-0238.2011.00127.x

Zott, K., Thibon, C., Bely, M., Lonvaud-Funel, A., Dubourdieu, D., and MasneufPomarede, I. (2011). The grape must non-Saccharomyces microbial community: impact on volatile thiol release. Int. J. Food Microbiol. 151, 210-215. doi: 10.1016/j.ijfoodmicro.2011.08.026

Conflict of Interest Statement: The authors declare that the research was conducted in the absence of any commercial or financial relationships that could be construed as a potential conflict of interest.

Copyright (c) 2016 Renault, Coulon, Moine, Thibon and Bely. This is an open-access article distributed under the terms of the Creative Commons Attribution License (CC BY). The use, distribution or reproduction in other forums is permitted, provided the original author(s) or licensor are credited and that the original publication in this journal is cited, in accordance with accepted academic practice. No use, distribution or reproduction is permitted which does not comply with these terms. 